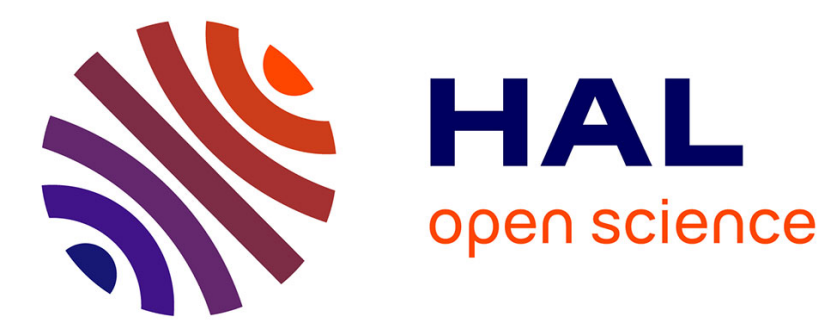

\title{
Deposition of hydrogenated silicon clusters for efficient epitaxial growth
}

\author{
Ha-Linh Thi Le, Fatme Jardali, Holger Vach
}

\section{To cite this version:}

Ha-Linh Thi Le, Fatme Jardali, Holger Vach. Deposition of hydrogenated silicon clusters for efficient epitaxial growth. Physical Chemistry Chemical Physics, 2018, 10.1039/c8cp00764k . hal-02395144

\section{HAL Id: hal-02395144 \\ https://hal.science/hal-02395144}

Submitted on 5 Dec 2019

HAL is a multi-disciplinary open access archive for the deposit and dissemination of scientific research documents, whether they are published or not. The documents may come from teaching and research institutions in France or abroad, or from public or private research centers.
L'archive ouverte pluridisciplinaire HAL, est destinée au dépôt et à la diffusion de documents scientifiques de niveau recherche, publiés ou non, émanant des établissements d'enseignement et de recherche français ou étrangers, des laboratoires publics ou privés. 


\title{
Journal Name
}

\section{ARTICLE TYPE}

Cite this: DOI: $10.1039 / x x x x x x x x x x$

\section{Deposition of hydrogenated silicon clusters for effi- cient epitaxial growth ${ }^{\dagger}$}

\author{
Ha-Linh Thi Le, Fatme Jardali, and Holger Vach*
}

Received Date

Accepted Date

DOI: $10.1039 / x x x x x x x x x x$

www.rsc.org/journalname

\begin{abstract}
Epitaxial silicon thin films grown from the deposition of plasma-born hydrogenated silicon nanoparticles using plasma enhanced chemical vapor deposition techniques have widely been investigated due to their potential applications in photovoltaic and nanoelectronic device technologies. However, the optimal experimental conditions and the underlying growth mechanisms leading to high-speed epitaxial growth of thin silicon films from hydrogenated silicon nanoparticles remain far from being understood. In the present work, extensive molecular dynamics simulations were performed to study the epitaxial growth of silicon thin films resulting from the deposition of plasma-born hydrogenated silicon clusters at low substrate temperatures under realistic reactor conditions. Strong evidence is presented that a temporary phase transition of the substrate area around the cluster impact site to the liquid state is necessary for the epitaxial growth to take place. We predict further that a non-normal incidence angle for the cluster impact significantly facilitates the epitaxial growth of thin crystalline silicon films.
\end{abstract}

\section{Introduction}

In the world of photovoltaic and nanoelectronic devices, epitaxially grown crystalline silicon (c-Si) thin films play an increasingly important role for technological improvement toward highefficient and low-cost products. ${ }^{1,2}$ New designs of solar cells, ultrafast transistors, efficient light emitting diodes, and photon detectors with minimal leakage currents are based on the use of intrinsic epitaxial silicon (epi-Si) absorbers, ${ }^{3}$ p-type epi-Si emitters, ${ }^{4}$ or n-type epi-Si emitters. ${ }^{5}$

Epitaxial silicon thin films can be grown on c-Si substrates with a huge variety of different deposition procedures. ${ }^{6}$ Plasma enhanced chemical vapor deposition (PECVD) presents many advantages over other techniques. The most important one is the fact that it is possible to obtain epitaxial silicon thin films at considerably lower substrate temperatures (as low as $150^{\circ} \mathrm{C}^{7}$ ) than other chemical vapor deposition (CVD) techniques such as atmospheric pressure CVD ${ }^{8}$ or hot wire CVD. ${ }^{9}$ When operated close to

CNRS, LPICM, Ecole Polytechnique, University Paris-Saclay, 91128 Palaiseau, France. Fax: +33 1693343 33; Tel: +33 1693343 73; E-mail: holger.vach@polytechnique.edu

$\dagger$ Electronic Supplementary Information (ESI) available: Two movies are provided to demonstrate the dynamics of cluster-induced epitaxial growth: one showing a typical example of the amorphous $\mathrm{Si}_{15} \mathrm{H}_{10}$ cluster approaching a H-terminated $\mathrm{Si}(100)$ $(2 \times 1)$ substrate at $373 \mathrm{~K}$ with an impact energy of $5.3 \mathrm{eV} /$ atom under normal incidence; and a second movie of the deposition of the crystalline $\mathrm{Si}_{29} \mathrm{H}_{24}$ cluster on a H-terminated $\mathrm{Si}(100)-(2 \times 1)$ substrate with an impact energy of $3.67 \mathrm{eV} /$ atom, a substrate temperature of $473 \mathrm{~K}$, and an incidence angle of 30 degrees; A description of the Ohira-Tersoff potential and its original and modified parameters is provided. See DOI: 10.1039/b000000x/ dusty plasma conditions, PECVD yields, in addition, significantly higher film growth rates in comparison to other low temperature techniques as Molecular Beam Epitaxy (MBE) due to the presence of plasma-born hydrogenated silicon nanoparticles. Those clusters do not only "catalyze" the film growth, but they also lead to films with high quality. ${ }^{10,11}$

Plasma synthesis of silicon nanoparticles has widely been studied to choose plasma conditions in such a way to precisely control their growth towards amorphous or crystalline structures. ${ }^{12-23}$ In addition, many aspects of the cluster-surface impact have been investigated with the goal to shed light on cluster-induced epitaxial growth of thin silicon films. ${ }^{24-36}$ Most previous studies only consider the interaction of pure silicon clusters and pristine silicon surfaces; ${ }^{24-32}$ a few investigations concerning the deposition of hydrogenated silicon clusters on H-terminated $\mathrm{Si}(100)$ $(2 \times 1)$ substrates under typical plasma conditions have been reported. ${ }^{35,36}$ Nevertheless, the underlying growth mechanisms for such epi-Si thin films have not been understood yet and there still remains a lot of controversy. ${ }^{25-27,29,33}$ Some conditions for such epitaxial growth have been qualitatively proposed; for instance, dissociation and spreading of impinging clusters, ${ }^{24,25}$ high surface diffusion, ${ }^{25}$ rearrangement of $\mathrm{Si}$ atoms caused by local heating at the impact point. ${ }^{26,29,32}$ Some authors suggest the need for cluster spreading 24,25 for epitaxial growth, while others exclude the importance of such a process. ${ }^{29,32}$ In a previous study, we have shown that silicon surfaces which were partly damaged by the violent impact of hydrogenated silicon clusters due to nonperfect epitaxial deposition conditions can be repaired to initial 
crystalline structures when they are exposed to appropriate flow rates of atomic hydrogen. ${ }^{37}$

In this work, we have carried out extensive molecular dynamics (MD) simulations to explore machenisms for the high-speed epitaxial growth of silicon thin films at low substrate temperatures from plasma-born hydrogenated silicon nanoparticles as experimentally observed in PECVD reactors. We demonstrate that a temporary phase transition of the substrate area around the cluster impact site to the liquid state is necessary for cluster-catalyzed epitaxy. Based on our simulations, we also provide some proposals to improve existing experimental setups for more efficient epitaxial growth.

\section{Computational method}

All MD simulations are carried out with the general chemical dynamics computer program VENUS. ${ }^{38}$ We employ the interatomic Ohira-Tersoff potential to simulate the cluster-surface interactions. ${ }^{39}$ Certain parameters were used here that have recently been optimized and tested thoroughly in comparison with $a b$ initio coupled-cluster calculations to model small silicon hydride systems. Details of the parameter optimization method are described elsewhere. ${ }^{40,41}$ The OT potential and its set of parameters are given in the Supplementary Information section. The time step used in our MD simulations is chosen to be 0.1 fs and each trajectory is followed for $200 \mathrm{ps}$. This value of the time step guarantees excellent energy conservation during the entire simulation time. With the aim towards a better understanding for the high-speed epitaxial growth of silicon thin films, we have used four hydrogenated silicon clusters with different sizes (very small, small, and moderate) and structures (amorphous and crystalline). The H-terminated $\mathrm{Si}(100)-(2 \times 1)$ surface is chosen as a substrate in our work since recent experimental studies have shown that epiSi thin films can be obtained on (100), but not on (111) c-Si wafers $^{11}$. With each impinging cluster, impact energy and substrate temperature are systematically examined since they are the most important parameters governing the cluster-surface deposition process $28,30,35,36$. The room temperature cluster is initially placed at a position where the cluster-surface interaction is fully negligible. After the addition of a center-of-mass velocity to all cluster atoms, the cluster approaches the surface with a given incidence angle. To avoid possible effects resulting from a precise impact location, we have generated and calculated over 20 trajectories with random rotational orientations and velocities of clusters sampled on a Boltzmann distribution around the indicated value for each impact condition.

Cluster preparation. We have employed plasma-born amorphous clusters $\left(\mathrm{Si}_{8} \mathrm{H}_{12}, \mathrm{Si}_{15} \mathrm{H}_{10}\right.$, and $\left.\mathrm{Si}_{28} \mathrm{H}_{13}\right)$ and the $1 \mathrm{~nm}$ hydrogenated silicon nanocrystal $\mathrm{Si}_{29} \mathrm{H}_{24}$ as impinging clusters. The three amorphous clusters were formed in a growth process under realistic plasma conditions as presented in Refs. [17] and [20]. The $\mathrm{Si}_{29} \mathrm{H}_{24}$ nanoparticle experimentally produced from the dispersion of bulk silicon by lateral electrochemical etching exhibits a crystalline structure and outstanding optical properties. ${ }^{42-45}$ We chose here the $\mathrm{Si}_{29} \mathrm{H}_{24}$ nanocrystal as a general model for crystalline nanoparticles generated in plasma reactors. All of these clusters were optimized to obtain their minimum energy struc- tures. Thereafter, the clusters were heated up to $300 \mathrm{~K}$ according to our experimental plasma conditions.

Surface preparation. The H-terminated $\mathrm{Si}(100)-(2 \times 1)$ substrate has the dimensions of $21.72 \AA \times 21.72 \AA \times 10.86 \AA$ and consists of $288 \mathrm{Si}$ atoms terminated by $32 \mathrm{H}$ atoms. Periodic boundary conditions are applied in the two directions parallel to the surface plane. The top silicon layer is a $(2 \times 1)$ reconstructed layer including silicon dimers. The bottom two silicon layers are kept rigid in their equilibrium positions to avoid translation and bending of the entire substrate in space. To control the substrate temperature, we apply a Berendsen thermostat ${ }^{46}$ to the three silicon layers above the two rigid layers. We heated up the substrate to 373,473 , and $573 \mathrm{~K}$ since those substrate temperatures are usually employed in our PECVD reactor.

Quantities to characterize structures and phase states. To analyze the structure characteristics of the clusters before the substrate impact and of the local impact region right after the impact and after cooling down to the initial substrate temperature, we have calculated their radial distribution functions (RDFs). The $\mathrm{RDF}$ gives the probability of finding a pair of atoms at a given interatomic distance and can be calculated as 47,48

$$
g(r)=\frac{n(r, \Delta r)}{\rho N \tau_{r u n} 4 \pi r^{2} \Delta r},
$$

where $g(r)$ is the RDF, $n(r, \Delta r)$ is the average number of atoms in a shell of width $\Delta r$ at a distance $r, \rho$ is the average atom density, $N$ is the total number of atoms, and $\tau_{\text {run }}$ is the number of steps selected to calculate the RDF.

In order to determine phase states of the local impact region right after the surface collision and after cooling down to the initial substrate temperature, we have also investigated the behavior of the mean-square displacement (MSD) defined as ${ }^{49}$

$$
\operatorname{MSD}(t)=\frac{1}{N}\left\langle\sum_{i=1}^{N}\left[\mathbf{r}_{i}\left(t_{0}+t\right)-\mathbf{r}_{i}\left(t_{0}\right)\right]^{2}\right\rangle
$$

where $\mathbf{r}_{i}(t)$ denotes the position of atom $i$ at time $t, N$ is the number of atoms.

All of the average values are calculated over the last 20 ps of each trajectory.

\section{Results and Discussions}

With the aim to search for the mechanisms leading to epitaxial growth of c-Si thin films from cluster deposition, we have first investigated the deposition of amorphous $\mathrm{Si}_{n} \mathrm{H}_{m}$ clusters since those more loosely bond impinging clusters were thought to be easier dissociated without serious damage of the substrate structure. ${ }^{36}$ The interaction of crystalline $\mathrm{Si}_{\mathrm{n}} \mathrm{H}_{\mathrm{m}}$ clusters with hydrogenated silicon surfaces was simulated to have a throughout understand of such an epitaxial growth.

\subsection{Deposition of amorphous $\mathrm{Si}_{\mathrm{n}} \mathrm{H}_{\mathrm{m}}$ clusters}

$\mathrm{Si}_{10} \mathrm{H}_{15}$ cluster. We have investigated the deposition mechanisms of $\mathrm{Si}_{15} \mathrm{H}_{10}$ cluster approached the substrate at $373 \mathrm{~K}$ under normal incidence with different impact energies up to $6 \mathrm{eV} /$ atom. Details of deposition mechanisms (from soft-landing to destruc- 
tive deposition) as a function of impact energy have been examined in Ref. [37]. For a typical example, we have observed epitaxial growth in the case where $\mathrm{Si}_{15} \mathrm{H}_{10}$ clusters approach the substrate with an impact energy of $5.3 \mathrm{eV} /$ atom. The Berendsen thermostat was switched of during the cluster deposition so that the system sampled in a microcanonical ensemble. After its impact, the cluster has completely penetrated into the substrate locally destroying the initial substrate lattice. Thereafter, the Si atoms start to reconstruct and eventually all cluster Si atoms adopt a perfect, epitaxial order on top of the initial substrate structure after about 80 ps. In the following, we define "local impact region" as the cluster Si atoms together with the substrate Si atoms touching the cluster. Directly after the impact, the temperature of the local impact region typically exceeds $3000 \mathrm{~K}$ due to the transfer of kinetic impact energy. Rapidly after, the temperature of this region cools down to $1600 \mathrm{~K}$. For the surface Si atoms surrounding the impact region, the same temperature is reached after a rapid heating from the initial substrate temperature of $373 \mathrm{~K}$ (see Fig. 1a and b). The local impact region and Si surface surrounding the impact region is then cooled down to $373 \mathrm{~K}$ by applying the Berendsen thermostat at the substrate with a coupling time constant of $1 \mathrm{fs}$. This value gives a reasonable temperature control rate and minimal disturbance of the physical behavior of the system.

To investigate the structure of the cluster before and after the substrate impact more quantitatively, we have calculated the radial distribution functions of the amorphous cluster at $300 \mathrm{~K}$ (before the impact), of the local impact region at $1 \mathrm{ps}$ after the impact and after cooling down to $373 \mathrm{~K}$; and compared them with the RDF of bulk c-Si at $373 \mathrm{~K}$ (see Fig. 2). In our calculation, the latter was simulated as a crystalline structure of $192 \mathrm{Si}$ atoms with three dimensional periodic boundary conditions at $373 \mathrm{~K}$. As can be seen in Fig. 2a, this c-Si structure is characterized by peaks that correspond to short- and long-range order. For the $\mathrm{Si}_{15} \mathrm{H}_{10}$ cluster and the local surface region of $29 \mathrm{Si}$ atoms, we focus on the first three peaks of the RDF. The disordered structure of the $\mathrm{Si}_{15} \mathrm{H}_{10}$ cluster and local surface region at 1 ps after the impact can be witnessed from the corresponding RDF, Fig. $2 \mathrm{~b}$ and $\mathrm{c}$. While the first peak in the case of the $\mathrm{Si}_{15} \mathrm{H}_{10}$ cluster exhibits a Si-Si interatomic distance is similar as the Si-Si bond length in bulk c-Si, the one in the case of the local surface region is broader. In both cases, the second peak is excessively broad and the third peak disappears in comparison to the RDF of bulk c-Si. In contrast, after the cooling down to $373 \mathrm{~K}$, Fig. 2d, all first three peaks of the RDF are consistent with those of bulk c-Si (at both absolute positions and relative intensities) indicating the crystalline structure of the local impact region.

The mean square displacement functions were calculated to compare the phase states of the cluster directly after the impact and after the cooling down to $373 \mathrm{~K}$. As can be seen in Fig. 3a and $b$, the MSD graphs of the local impact region indicate a liquid state at 1 ps after the impact and a solid state after the cooling down to $373 \mathrm{~K}$.

This observation raises the question whether the impact energy per atom (5.3 eV/atom) or the total kinetic energy $(79.5 \mathrm{eV})$ is important and necessary to obtain epitaxial growth of thin sili-
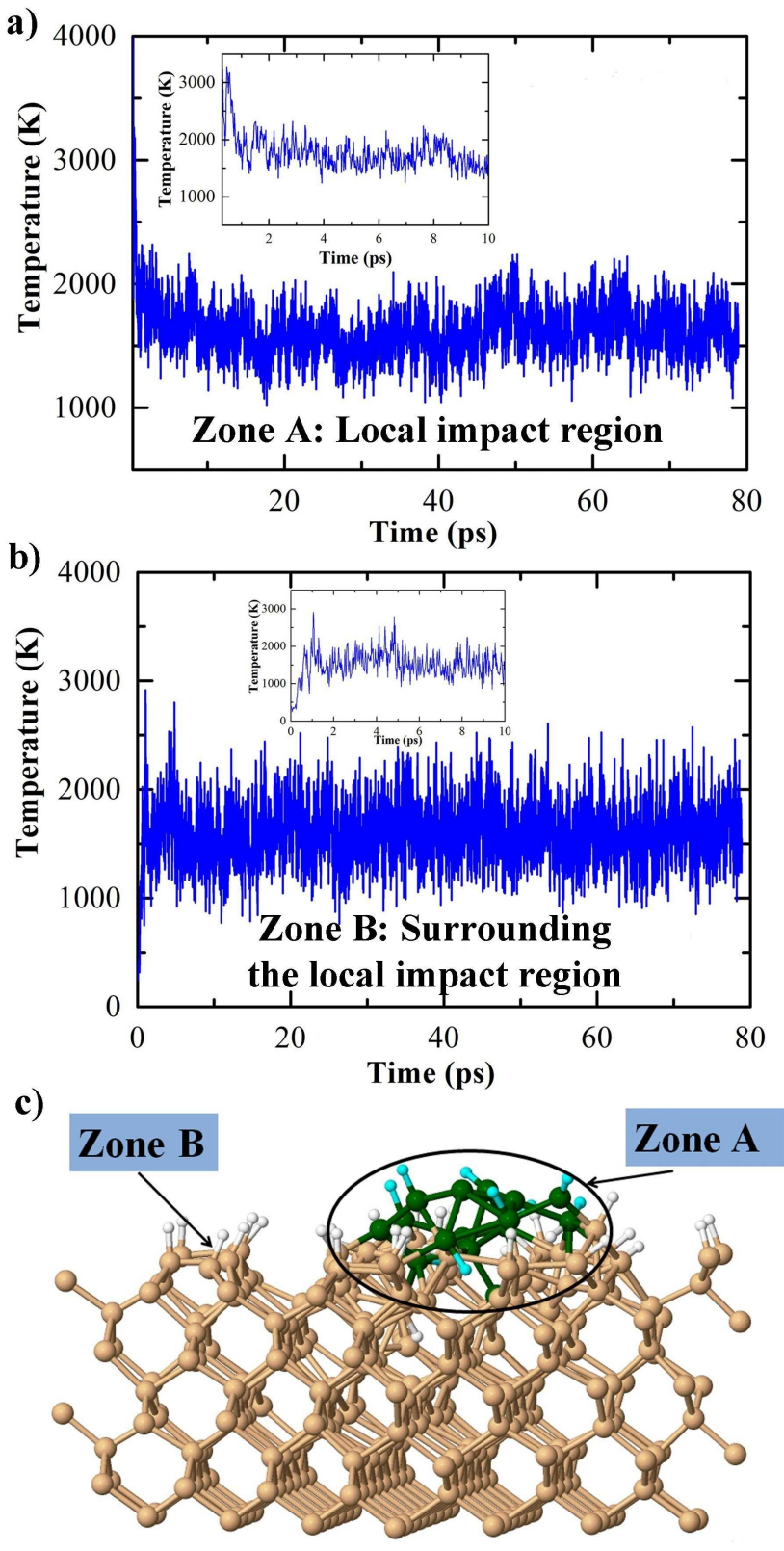

Fig. 1 a) and b) Time evolution of the instantaneous temperature of: local impact region (zone $\mathrm{A}$ ) and substrate $\mathrm{Si}$ atoms surrounding the impact region (zone $\mathrm{B}$ ), respectively. The initial substrate temperature was 373 $\mathrm{K}$ and the $\mathrm{Si}_{15} \mathrm{H}_{10}$ cluster approached the substrate at normal incidence with an impact energy of $5.3 \mathrm{eV} /$ atom. c) Schematic representation of zones $\mathrm{A}$ and $\mathrm{B}$. The large brown spheres represent surface $\mathrm{Si}$ atoms while the green ones represent $\mathrm{Si}$ atoms of the $\mathrm{Si}_{15} \mathrm{H}_{10}$ cluster. The small white spheres represent surface $\mathrm{H}$ atoms while the cyan ones represent $\mathrm{H}$ atoms of the $\mathrm{Si}_{15} \mathrm{H}_{10}$ cluster. 

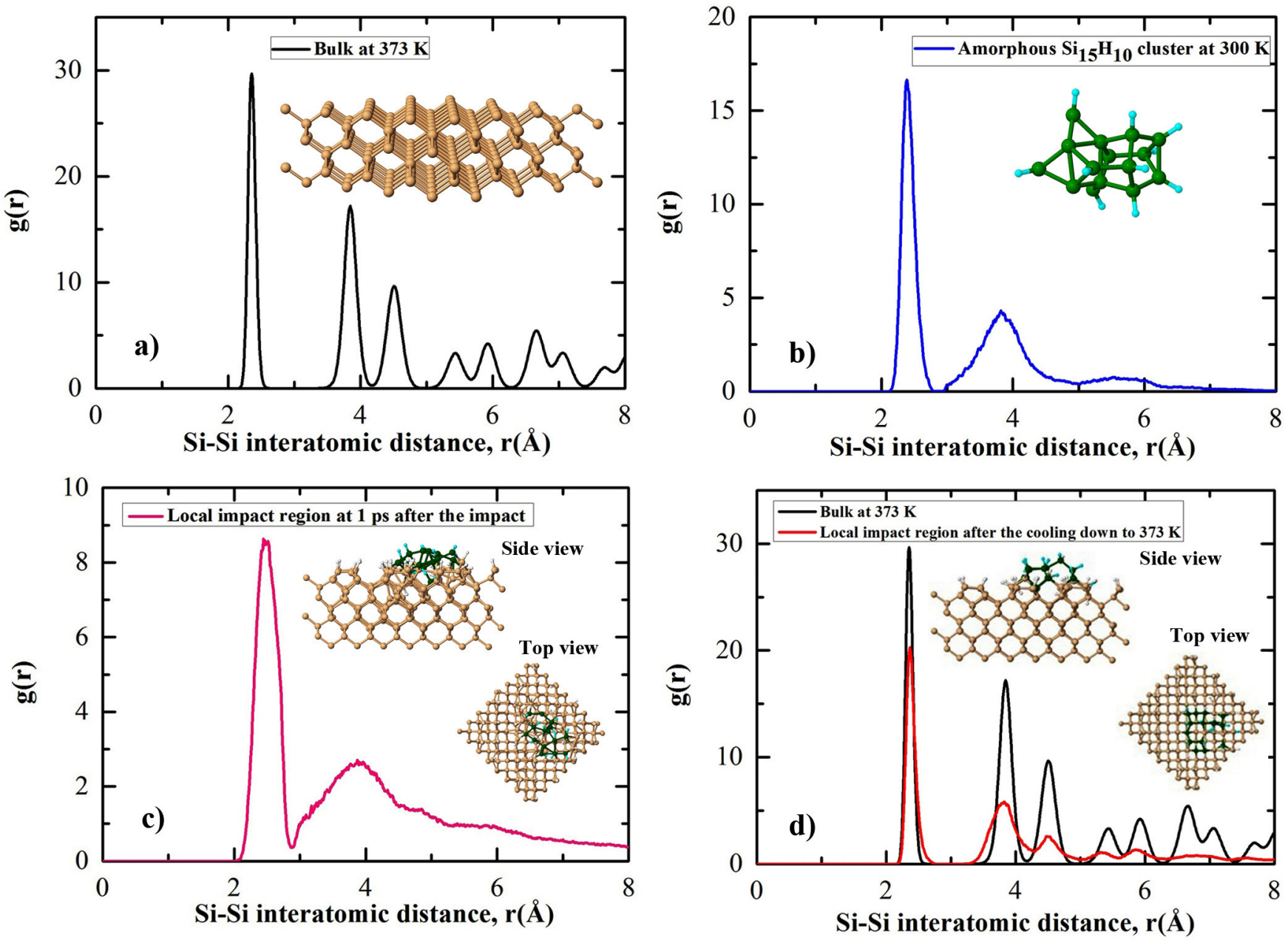

Fig. 2 Radial distribution functions of: a) bulk silicon at $373 \mathrm{~K}$, b) the amorphous $\mathrm{Si}_{15} \mathrm{H}_{10}$ cluster at $300 \mathrm{~K}$ before impact, c) local impact region at 1 ps after the impact, and d) local impact region after the cooling down to $373 \mathrm{~K}$. The impact energy was $5.3 \mathrm{eV} /$ atom. The RDF of local impact region after the cooling down to $373 \mathrm{~K}$ is consistent with that of bulk c-Si (see text).
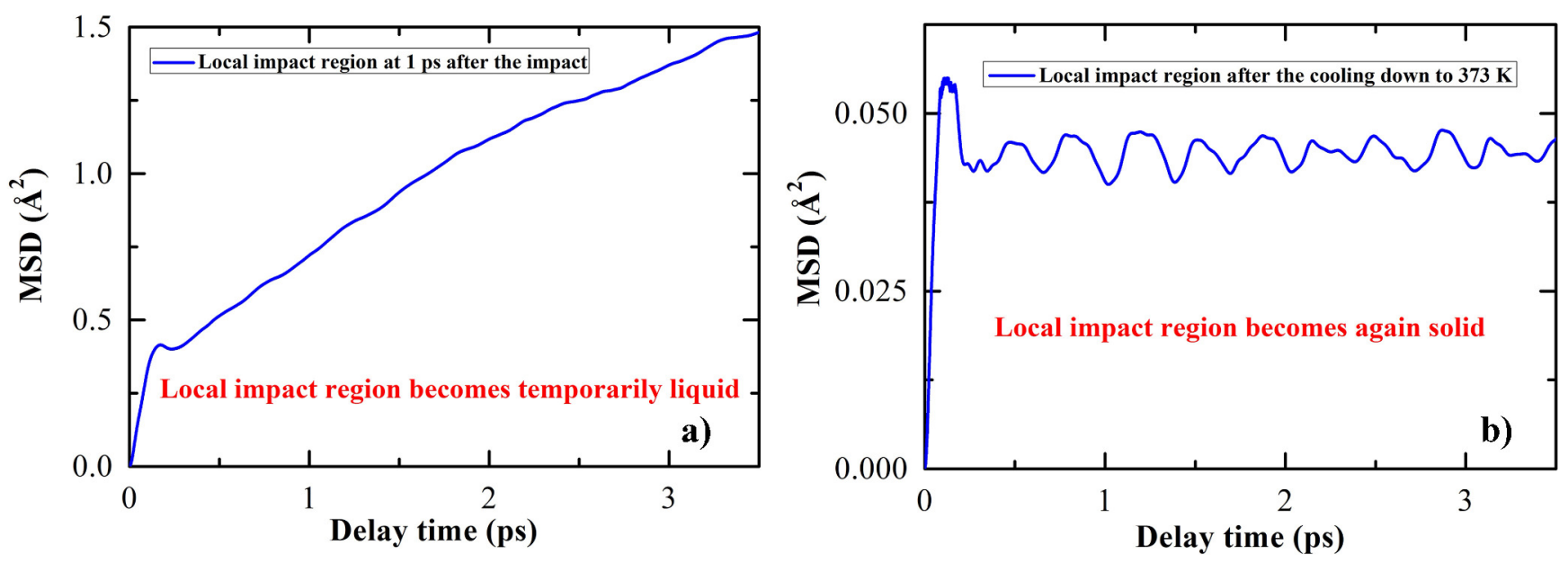

Fig. 3 Mean square displacement of the Si atoms in the local impact region: a) at 1 ps after the impact and b) after the cooling down to $373 \mathrm{~K}$. The $\mathrm{Si}_{15} \mathrm{H}_{10}$ cluster approached the substrate at normal incidence with an impact energy of $5.3 \mathrm{eV} /$ atom. 
con films by means of cluster deposition. Therefore, the deposition processes of smaller and bigger hydrogenated silicon clusters with both amorphous and crystalline structures were investigated in order to find the crucial experimental parameters that govern such an epitaxial growth.

$\mathrm{Si}_{8} \mathrm{H}_{12}$ cluster. We studied the $\mathrm{Si}_{8} \mathrm{H}_{12}$ cluster-surface deposition processes with two impact energies (5.3 and $9.95 \mathrm{eV} /$ atom; the latter corresponding to a total kinetic energy of $79.5 \mathrm{eV}$ ) and a normal incidence angle. And to investigate the influence of substrate temperature on epitaxial growth, we heated up the substrate to 373,473 , and $573 \mathrm{~K}$. We counted the number of Si atoms found in epitaxial positions and define the epitaxial efficiency as the ratio of the number of Si atoms in epitaxial positions and the number of Si atoms in the impinging cluster. Here, we consider a Si atom to be in an epitaxial position when its presence increases the volume of the crystalline part of the $\mathrm{Si}(100)$ surface.

The results shown in Table 1 reveal that for the $\mathrm{Si}_{8} \mathrm{H}_{12}$ cluster a total kinetic energy of about $79.5 \mathrm{eV}$ plays a more important role than the impact energy per atom for the epitaxial growth. An impact energy of about $5.3 \mathrm{eV} /$ atom leading to a total kinetic energy of only $42 \mathrm{eV}$ results in a temperature of the local impact region smaller than $1220 \mathrm{~K}$, whereas this temperature is considerably higher in the case of total kinetic energy of about $79.5 \mathrm{eV}$ for all three investigated substrate temperatures (see Table 1 ). Therefore, the impact energy of about $5.3 \mathrm{eV} /$ atom is apparently not high enough to access epitaxial positions for all cluster Si atoms. Moreover, higher substrate temperatures are not always a warranty for better epitaxy. That means we have to look at the same time at both impact energy and substrate temperature. Consulting again the MSDs of the local impact region, we see that only the total kinetic energy of about $79.5 \mathrm{eV}$ permits the transition to the liquid state for all three investigated substrate temperatures. Therefore, we suggest that a temporary phase transition to the liquid state of the local impact region is necessary for the clustercatalyzed epitaxial growth to take place most efficiently.

$\mathrm{Si}_{28} \mathrm{H}_{13}$ cluster. The deposition of a considerably larger amorphous cluster $\left(\mathrm{Si}_{28} \mathrm{H}_{13}\right)$ was simulated to confirm our conclusion that it is the total kinetic energy of $79.5 \mathrm{eV}$ and not the impact energy per atom of $5.3 \mathrm{eV}$ that is necessary to achieve epitaxial growth and to test our suggestion for the need of a temporary liquid state. To this end, we performed two simulation series.

First, we deposited the $\mathrm{Si}_{28} \mathrm{H}_{13}$ clusters under normal incidence on H-terminated $\mathrm{Si}(100)-(2 \times 1)$ surfaces at a substrate temperature of $373 \mathrm{~K}$ and changed the impact energies. The epitaxial efficiency is shown in Fig. 4. For the case of total kinetic energy of about $79.5 \mathrm{eV}$ (i.e. an impact energy of $2.84 \mathrm{eV} / \mathrm{atom}$ ), we find an epitaxial efficiency of $75 \%$ while for an impact energy of 5.37 $\mathrm{eV} /$ atom, we find a seriously damaged surface and etching due to the extremely high resulting temperature of the local impact region of about $5000 \mathrm{~K}$ that still remains as high as $2250 \mathrm{~K}$ after the initial equilibration. Even after slowly cooling down to room temperature, we find the surface as damaged and no hint for a possible recovery to the initial structure could be detected. This observation again suggests that it is the total kinetic energy that is responsible for epitaxy.

Second, the $\mathrm{Si}_{28} \mathrm{H}_{13}$ clusters were deposited again under nor- mal incidence on H-terminated $\operatorname{Si}(100)-(2 \times 1)$ surfaces with an impact energy of $2.84 \mathrm{eV} /$ atom, but at two different substrate temperatures of 373 and $573 \mathrm{~K}$. The structural evolution of the local impact area during the deposition processes for the two cases are quite similar. This observation is consistent with an earlier investigation. ${ }^{35}$ As observed in the case of the $\mathrm{Si}_{8} \mathrm{H}_{12}$ cluster, we find again a decrease in epitaxial efficiency for the higher substrate temperature; namely, 75 and $64 \%$ for substrate temperatures of 373 and $573 \mathrm{~K}$, respectively.

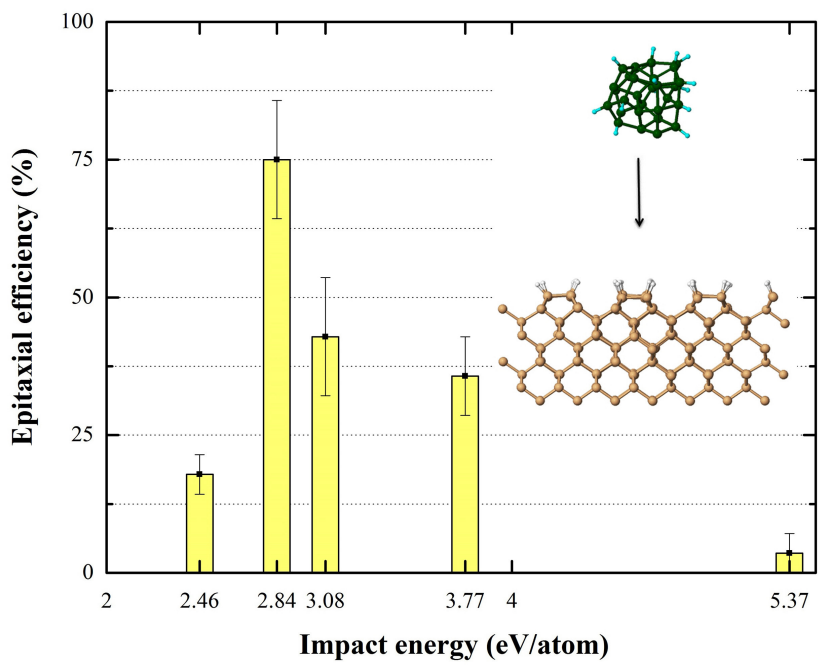

Fig. 4 Epitaxial efficiency for the deposition of amorphous $\mathrm{Si}_{28} \mathrm{H}_{13}$ clusters on $\mathrm{H}$-terminated $\mathrm{Si}(100)-(2 \times 1)$ surfaces with a normal incidence angle and various impact energies. The substrate temperature is always $373 \mathrm{~K}$.

Our investigation with all three employed clusters indicates that the epitaxial efficiency depends most crucially on the total kinetic energy of the impacting clusters. Therefore, we propose for the experimentalists to control the velocity of the impacting clusters to be in the right energy window to allow for the necessary phase transition to the liquid state, but to avoid too fast impinging clusters that damage the substrate too extensively. To this end, a biased grid in front of the substrate could be used to control the impact energy better.

\subsection{Deposition of crystalline $\mathrm{Si}_{\mathrm{n}} \mathrm{H}_{\mathrm{m}}$ clusters}

In the preceding, we have focused on the deposition of amorphous $\mathrm{Si}_{\mathrm{n}} \mathrm{H}_{\mathrm{m}}$ clusters. As a result, we conclude that a temporary phase transition to the liquid state is necessary for cluster-induced epitaxial growth. In the following, we like to explore the role of the cluster structure for epitaxial growth. To this end, the crystalline $\mathrm{Si}_{29} \mathrm{H}_{24}$ cluster is deposited under normal incidence on $\mathrm{H}$ terminated $\mathrm{Si}(100)-(2 \times 1)$ surfaces.

First, the impact energies are adjusted and the substrate temperature is always $373 \mathrm{~K}$. The resulting epitaxial efficiencies can be seen in Fig. 5. Using the total energy criterion (i.e., an impact energy of about $2.74 \mathrm{eV} /$ atom in this case), we get a relatively low efficiency of only $17 \%$, while for the impact energy per atom criterion (impact energy of $5.31 \mathrm{eV} /$ atom), we find again a seriously damaged surface and extensive etching. A considerably 
Table 1 Table of epitaxial efficiency for the deposition of $\mathrm{Si}_{8} \mathrm{H}_{12}$ clusters on $\mathrm{H}$-terminated $\mathrm{Si}(100)-(2 \times 1)$ substrates with a normal incidence angle, various impact energies and substrate temperatures

\begin{tabular}{llll}
\hline Impact energy (eV/atom) & Substrate temperature (K) & Epitaxial efficiency (\%) & Temperature of local impact region (K) \\
\hline \multirow{2}{*}{5.3} & 373 & 37.5 & 990 \\
& 473 & 37.5 & 1050 \\
& 573 & 75 & 1220 \\
\hline \multirow{2}{*}{9.95} & 373 & 100 & 1540 \\
& 473 & 100 & 1620 \\
& 573 & 25 & 1720 \\
\hline
\end{tabular}

higher efficiency is obtained when we slightly increase the impact energy to $3.03 \mathrm{eV} /$ atom. Tentatively, we attribute this observation to the fact that we need more energy to melt a crystalline cluster than an amorphous one.

Second, all $\mathrm{Si}_{29} \mathrm{H}_{24}$ clusters have a total impact energy of 79.5 $\mathrm{eV}$ and the substrate temperature is altered. There is no significant difference in the structural evolution of the local impact area during the deposition processes for the three investigated substrate temperatures $(373,473$, and $573 \mathrm{~K})$. The corresponding epitaxial efficiencies are 17,14 , and $31 \%$, respectively.

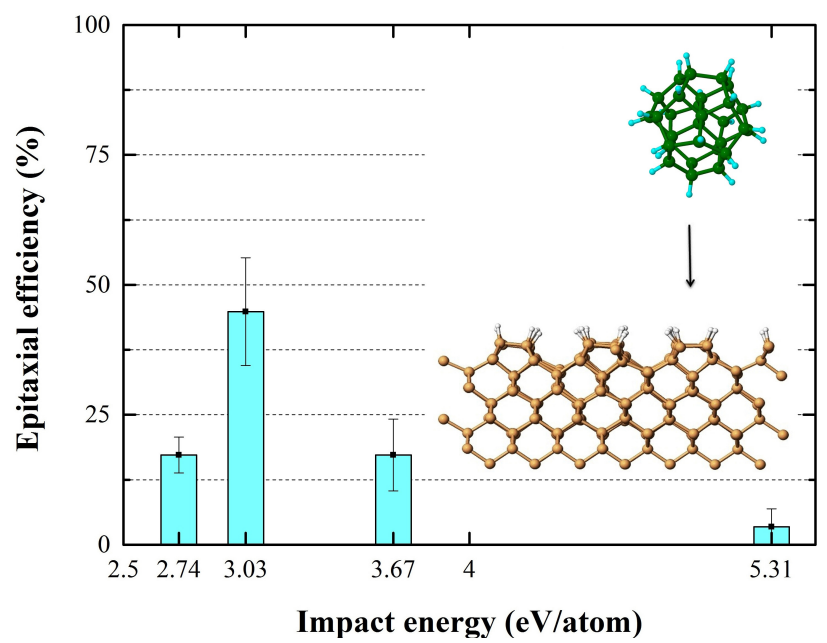

Fig. 5 Epitaxial efficiency for the deposition of $\mathrm{Si}_{29} \mathrm{H}_{24}$ clusters on $\mathrm{H}$ terminated $\mathrm{Si}(100)-(2 \times 1)$ substrates with a normal incidence angle and various impact energies. The substrate temperature is always $373 \mathrm{~K}$.

Whatever combination of incident kinetic energy and substrate temperature was employed for the crystalline $\mathrm{Si}_{29} \mathrm{H}_{24}$ cluster, we could never observe a case of complete epitaxial growth. The epitaxial growth efficiency is actually quite low in comparison to our studies with amorphous clusters. Up to now, however, we have only investigated normal incidence impact conditions. In those simulations, we have often observed that the top atoms of the cluster remain far away from the substrate atoms preventing them from finding epitaxial positions. Therefore, we were wondering if a non-normal incidence angle could not help to spread the cluster out over the substrate permitting more cluster atoms to be in direct contact with the substrate. This idea was triggered by previous studies concerning the surface scattering of large rare gas clusters. ${ }^{50-52}$ To this end, we deposited the crystalline $\mathrm{Si}_{29} \mathrm{H}_{24}$ clusters on H-terminated $\mathrm{Si}(100)-(2 \times 1)$ surfaces with an impact energy of $3.67 \mathrm{eV} /$ atom, a substrate temperature of $473 \mathrm{~K}$, and different incidence angles from 0 to 55 degrees as measured from the surface normal direction.

The effect of incidence angle on the epitaxial efficiency is shown in Fig. 6. Keeping the same deposition conditions of total kinetic impact energy and substrate temperature, we see that the epitaxial efficiency increases in all cases where non-normal incidence angles are used; i.e, a non-normal incidence angle significantly facilitates the epitaxial growth. An increase of epitaxial efficiency by a factor of four has been found for an incidence angle of only 30 degrees. It would be appropriate to mention that the obtained incidence angle (i.e. 30 degrees) might be an optimum angle for the considered cluster size range. If the angle is too close to the normal (i.e. too small), the atoms located on top of the cluster do not get close enough to the substrate atoms and might not participate in the collective melting. If, however, the angle is too close to the grazing incidence angle (i.e. too large), there is not enough normal kinetic energy left for the phase transition to take place. Therefore, an ideal incidence angle should depend on the precise deposition parameter as, for instance, the cluster size.

Although it appears like a major experimental challenge to choose only one precise impact angle (as for instance 30 degrees) considering the random flight direction of clusters in the plasma, we would like to suggest the following ideas to control the deposition of clusters under a given incident angle: We can imagine the use of two subsequent "pinholes" that define a precise normal direction of the impinging clusters and then tilt the substrate to the desired angle. Such a setup, however, would seriously jeopardize the maximum deposition rate. Alternatively, one might want to take advantage of the fact that $75 \%$ of the clusters leaving the plasma are positively charged. ${ }^{53}$ Consequently, the use of an electrically biased grid placed in front of the substrate should permit us to have most clusters arrive at a quasi-normal direction to the substrate. The simple tilting of the substrate to a desired impact angle in this case, however, might result in a strong perturbation of the electric field lines, which might not lead to the desired result. Therefore, we propose, in addition, to apply a set of two horizontal electrodes installed on both sides above the substrate that will allow us to add a rather precisely defined impact velocity component parallel to the substrate. The simultaneous choice of the voltages applied to the grid and to the parallel electrodes will then permit us to control both the normal component of the impact energy; i.e., the part that controls the amount of cluster heating, and the cluster impact angle; i.e., the amount of the 
cluster spreading and flattening on the substrate. Reversing the polarity at half of the deposition time can assure the deposition on the entire substrate.

Finally, for both amorphous and crystalline clusters, the clusters partially or totally penetrate into the substrate lattice. Cluster atoms and local substrate atoms around the impact region then form a disordered structure. Epitaxial growth may occur at impact energies that are high enough to allow the local impact region to become temporary liquid. During an intermediate transition period, the Si atoms of the cluster and of the local surface become indistinguishable. Subsequently, those Si atoms rearrange themselves. Some of the cluster $\mathrm{Si}$ atoms might insert themselves into lattice positions, whereas some $\mathrm{Si}$ atoms of the initial bulk part of the substrate might find themselves on top in the new epitaxially grown layer. After the cluster-substrate impact, some of the $\mathrm{H}$ atoms of the cluster and the surface may be trapped deeply inside the substrate, around and under the cluster impact site. Following the epitaxial growth on a longer timescale, these $\mathrm{H}$ atoms diffuse inside the substrate and ultimately move toward the surface.

We have simulated many trajectories and our MSD results show that whenever there was epitaxial growth, it was preceded by a liquid state phase. Therefore, we conclude that a temporary phase transition to the liquid state is necessary for the clustercatalyzed epitaxial growth of silicon thin films. This condition is crucial because there are many different structures involved: the incoming clusters are either amorphous or crystalline; in the latter case, their precise structure depends on their size. In addition, the structure of the substrate surface is quite different from the one of the substrate bulk. After the impact, the atoms formerly belonging either to the cluster or to the surface of the substrate might become part of the substrate bulk and vice versa. Therefore, a temporary lost of all structural information and a subsequent recrystallization of all involved atoms appears, thus, to be the most universal and natural way for the efficient formation of a perfect epitaxial layer.

It is worth noting that the realism of our study of individual cluster impacts is warrant by the fact that the epitaxial deposition dynamics of a given cluster will be finished a long time before the next cluster will arrive on the substrate. Since the deposition rate of epi-Si films is $1.5-2 \AA / \mathrm{s}^{54}$ and the distance of two consecutive Si layers is about $1.358 \AA$, we should expect that the deposition of two Si monolayers takes about one second. This value is roughly six orders of magnitude longer than the epitaxial deposition dynamics duration of a given $\mathrm{Si}$ cluster; i.e., the process is terminated a long time before a second cluster can possibly arrive in the same part of the surface and undergo a quite similar deposition dynamics.

\section{Conclusions}

Epitaxial growth of c-Si thin films induced by the deposition of hydrogenated silicon clusters is a complicated process. The epitaxial efficiency depends on the combination of all investigated parameters: impact energy, substrate temperature, incidence angle, cluster size and structure. For the two smallest investigated impinging clusters: A temporary phase transition to the liquid

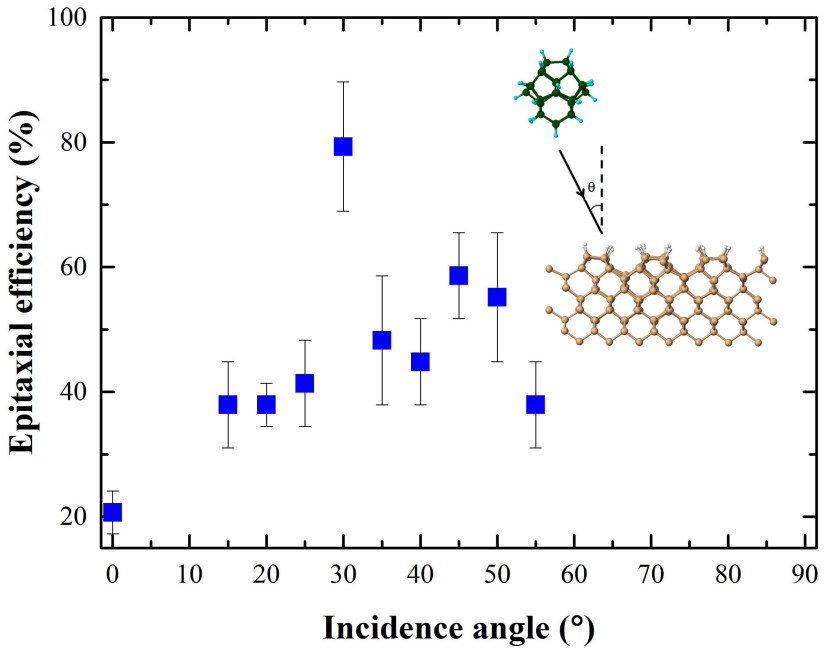

Fig. 6 Epitaxial efficiency for the deposition of $\mathrm{Si}_{29} \mathrm{H}_{24}$ clusters on $\mathrm{H}$-terminated $\mathrm{Si}(100)-(2 \times 1)$ substrates with an impact energy of 3.67 $\mathrm{eV} /$ atom, a substrate temperature of $473 \mathrm{~K}$, and various incidence angles.

state is necessary and sufficient for the cluster-catalyzed epitaxial growth of silicon thin films. For the larger clusters: In addition to the liquid state transition, the introduction of a non-normal incidence angle appears to be necessary to spread out the cluster on the substrate to permit a majority of the cluster atoms to get close enough to potentially epitaxial positions of the substrate. We tentatively suggest that the need for non-normal incidence increases with the size of the impinging clusters.

In principle, the local phase transition to the liquid state can experimentally be observed by a drastic change in optical reflectivity since liquid silicon is a metal. However, such an experiment presents a true challenge since the liquid state only lasts for about $10 \mathrm{ps}$, has a diameter of a few $\mathrm{nm}$, and can occur anywhere on the wafer. The presented new insight into the mechanism of clustercatalysed epitaxy might lead to some immediate applications for industrial production of high quality thin c-Si films at low temperatures with high-speed deposition rates for photovoltaic and nanoelectronic devices.

\section{Acknowledgments}

The discussions about the VENUS code with Prof. Gilles Peslherbe and Dr. Ning Ning are highly appriciated. We acknowledge the HPC Centers IDRIS (Grant i2017-090642) and CERMM for computational resources. We are also grateful to the Vietnamese Government (USTH project) for the scholarship awarded to HLTL, the Hariri Foundation for Sustainable Human Development for the scholarship awarded to F.J., the EADS Corporate Foundation, and the Conseil franco-québécois de coopération universitaire for the financial support.

\section{References}

1 J. Poortmans and V. Arkhipov, Thin Film Solar Cells Fabrication, Characterization and Applications, John Wiley \& Son Ltd, West Sussex, 2006, p. 1.

2 J. Yoo, S. A. Dayeh, N. C. Bartelt, W. Tang, A. T. Findikoglu 
and S. T. Picraux, Nano Lett., 2014, 14, 6121-6124.

3 R. Cariou, R. Ruggeri, P. Chatterjee, J.-L. Gentner and P. Roca i Cabarrocas, SPIE optics and photonics, San Diego : United States, 2012, 84700B, 8470.

4 J. Damon-Lacoste, M. Labrune, S. Granata, D. Daineka and P. Roca i Cabarrocas, author, Honolulu, HI, 2010, pp. 001352001357.

5 E. Schmich, H. Lautenschlager, T. Frieß, F. Trenkle, N. Schillinger and S. Reber, Journal of Crystal Growth, 2008, 16, 159-170.

6 M. Moreno, G. Patriarche and P. Roca i Cabarrocas, Journal of materials research, 2013, 28, 1626-1632.

7 D. Shahrjerdi, B. Hekmatshoar, S. W. Bedell, M. Hopstaken and D. K. Sadana, Journal of Electronic Materials, 2011, 41, 494-497.

8 E. Schmich, N. Schillinger and S. Reber, Surf. \& Coat. Tech., 2007, 201, 9325-9329.

9 C. W. Teplin, K. Alberi, M. Shub, C. Beall, I. T. Martin, M. J. Romero, D. L. Young, R. C. Reedy, P. Stradins and H. M. Branz, Appl. Phys. Lett., 2010, 96, 201901.

10 P. Roca i Cabarrocas, K.-H. Kim, R. Cariou, M. Labrune, E. V. Johnson, M. Moreno, A. T. Rio, S. Abolmasov and S. Kasouit, MRS Online Proceedings Library, 2012, 1426, 319-329.

11 P. Roca i Cabarrocas, R. Cariou and M. Labrune, Journal of Non-Crystalline Solids, 2012, 358, 2000-2003.

12 R. M. Sankaran, D. Holunga, R. C. Flagan and K. P. Giapis, Nano Lett., 2005, 5, 537-541.

13 M. Cavarroc, M. Mikikian, G. Perrier and L. Boufendi, Appl. Phys. Lett., 2006, 89, 013107.

14 T. Nguyen-Tran, P. Roca i Cabarrocas and G. Patriarche, Appl. Phys. Lett., 2007, 91, 111501.

15 S. Askari, , I. Levchenko, K. Ostrikov, P. Maguire and D. Mariotti, Appl. Phys. Lett., 2014, 104, 163103.

16 B. Barwe, A. Stein, O. E. Cibulka, I. Pelant, J. Ghanbaja, T. Belmonte and J. Benedikt, Plasma Processes and Polymers, 2015, 12, 132-140.

17 H. Vach and Q. Brulin, Phys. Rev. Lett., 2005, 95, 165502.

18 H. Vach, Nano Lett., 2011, 11, 5477-5481.

19 H. Vach, J. Chem. Theory Comput., 2012, 8, 2088-2094.

20 N. C. Forero-Martinez, H.-L. T. Le and H. Vach, Plasma Chemistry and Plasma Processing, 2014, 34, 535-543.

21 H. Vach, Phys. Rev. Lett., 2014, 112, 197401.

22 H. Vach, Chem. Phys. Lett., 2014, 614, 199-203.

23 H. Vach, L. V. Ivanova, Q. K. Timerghazin, F. Jardali and H.L. T. Le, Journal of Computational Chemistry, 2015, 36, 20892094.

24 C. M. S. R. Biswas, G. S. Grest, Phys. Rev. B, 1988, 38, 81548162.

25 I. Kwon, R. Biswas, G. S. Grest and C. M. Soukoulis, Phys. Rev. $B, 1990,41,3678-3687$.

26 G. Gilmer, C. Roland, D. Stock, M. Jaraiz and T. D. de la Rubia, Mater. Sci. and Eng. B, 1996, 37, 1-7.

27 J. Q. Xie and J. Y. Feng, Nucl. Instr. and Methods in Phys. Re- search $B, 1998,142,77-82$.

28 J. Q. Xie, J. Y. Feng and H. W. Lu, Modelling Simul. Mater. Sci. Eng., 1999, 7, 289-295.

29 J. Tarus and K. Nordlund, Nucl. Instr. and Methods in Phys. Research B, 2003, 212, 281-285.

30 Mazzone, Journal of Computer-Aided Materials Design, 2002, 9, 1-9.

31 Mazzone, Nucl. Instr. and Methods in Phys. Research B, 2002, 196, 51-60.

32 L. W. Chen, Y. Shibuta, M. Kambara and T. Yoshida, J. Appl. Phys., 2012, 111, 123301.

33 L. W. Chen, Y. Shibuta, M. Kambara and T. Yoshida, J. Phys. D: Appl. Phys., 2013, 46, 425302.

34 D. B. Graves and P. Brault, J. Phys. D: Appl. Phys., 2009, 42, 194011.

35 N. Ning, S. M. Rinaldi and H. Vach, Thin Solid Films, 2009, 517, 6234-6238.

36 N. Ning and H. Vach, J. Phys. Chem. A, 2010, 114, 3297-3305.

37 H.-L. T. Le, N. C. Forero-Martinez and H. Vach, Chem. Phys. Lett., 2014, 610-611, 223.

38 W. L. Hase, R. J. Duchovic, X. Hu, A. Komornicki, K. F. Lim, D. Lu, G. H. Peslherbe, K. N. Swamy, S. R. V. Linde, A. Varanda, W. Haobin and R. J. Wolf, 1996, (Department of Chemistry - Wayne State University, Detroit, MI, 1996), year.

39 T. Ohira, O. Ukai and M. Noda, Surf. Sci., 2000, 458, 216228.

40 N. Ning, G. Dolgonos, W. Morscheidt, A. Michau, K. Hassouni, and H. Vach, Comput. Methods Sci. Eng., 2007, 1, 224-249.

41 N. Ning, Ph.D. thesis, Ecole Polytechnique, 2008.

42 L. Mitas, J. Therrien, R. Twesten, G. Belomoin and M. H. Nayfeh, Appl. Phys. Lett., 2001, 78, 1918-1920.

43 G. Belomoin, E. Rogozhina, J. Therrien, P. V. Braun, L. Abuhassan, M. H. Nayfeh, L. Wagner and L. Mitas, Phys. Rev. B, 2002, 65, 193406.

44 S. Rao, J. Sutin, R. Clegg, E. Gratton, , M. H. Nayfeh, S. Habbal, A. Tsolakidis and R. M. Martin, Phys. Rev. B, 2004, 69, 205319.

45 S. Rao, K. Mantey, J. Therrien, A. Smith and M. H. Nayfeh, Phys. Rev. B, 2007, 76, 155316.1-155316.9.

46 H. J. C. Berendsen, J. P. M. Postma, W. F. Vangunsteren, A. Dinola and J. R. Haak, J. Chem. Phys., 1984, 81, 3684-3690.

47 M. P. Allen and D. J. Tildesley, Computer simulations of liquids, Clarendon Press, Oxford, 1987, p. 54 and 183.

48 C. D. Laboratory, Democritus: The Radial Distribution Function, October, 2013, http://www.compsoc.man.ac.uk/ lucky/Democritus/Theory/rdf.html.

49 M. P. Allen and D. J. Tildesley, Computer simulations of liquids, Clarendon Press, Oxford, 1987, p. 58 and 185.

50 H. Vach, Phys. Rev. B, 2000, 61, 2310-2315.

51 E. Fort, A. D. Martino, F. Pradère, M. Châtelet and H. Vach, J. Chem. Phys., 1999, 110, 2579-2587.

52 F. Pradère, M. Benslimane, M. Châtelet, A. D. Martino and H. Vach, Surf. Sci., 1997, 375, 375. 
53 N. Chaâbane, V. Suendo, H. Vach, and P. R. i Cabarrocasa, Appl. Phys. Lett., 2006, 88, 203111.
54 R. Cariou, M. Labrune and P. R. i Cabarrocas, Solar Energy Materials and Solar Cells, 2011, 95, 2260-2263. 